\title{
INCIDENCIA DE PATOLOGÍA/PRECANCEROSA GINECOLÓGICA EN EL CENTRO DETECTOR/DEL CÁNCER DE TACNA, 1984 - 1993
}

Salvador Moarri Hoss ${ }^{1}$, Rina Alvarez Becerra ${ }^{2}$

\author{
R E S U M E N
}

En el Peri, el cáncer de cuello uterino es la primera causa de muerte por cáncer en la mujer, siendo la tasa de mortalidad de 1980 por 100,000 habitantes por año para las mujeres mayores de 15 años.

El Papanicolaou es un examen citologico de bajo costo y que puede permitir además de la prevención de cáncer, el tratamiento de infecciones que son detectadas tanto por examen como por el estudio de papanicolaou.

En el departamento de Tacna, la Liga Peruana de Lucha contra el Cäncer se fundo con la finalidad de prevenir el cáncer en la población tacneña. La siguiente es una revisión de 10 años que nos arroja interesantes datos.

\section{A B S TRACT}

In Perú, uterine cervix is the first cause of cancer death in women, being the dead rate: 1,980 of 100,000 inhabitants per year, for women older than 15 years.

Papanicolau is a cheap cytological test that permits to prevent cancer as well as treatment of infections also detected by other clinical tests.

In Tacna, Perú, La Liga Peruana de Lucha contra el Cáncer (Peruvian league against cancer), was founded with the goal of preventing cancer in female population in Tacna.

A study of the last 10 years (from 198 to 199) gives interesting data about this topic.

Statistics were taken from files of La Liga Peruana de Lucha contra el Cáncer in Tacna.

\section{INTRODUCCIÓN}

- El cáncer de cuello uterino es un tumor maligno que se origina en el orificio externo del cérvix (zona de transformación), y que va a completar gradualmente esta parte del útero, para extenderse a los parametros, cuerpo, vagina, hasta llegar al hueso pélvico e infiltrarse en los órganos vecinos (vejiga, recto) y finalmente dar metástasis a distancia.

1. Médico.

2. Licenciada en obstetricia.
Cabe agregar que al elevado riesgo de enfermar y morir por este mal, se suman los efectos de tipo psicosocial, ya que esta dolencia es más frecuente en una etapa en que las mujeres son núcleo del grupo familiar.

Al desequilibrio que ocasiona el mal, se agrega la actitud de rechazo por la pareja, que en muchos casos termina con la desintegración de la familia.

Todas estas consideraciones nos han motivado a llevar adelante este estudio, sobre que un gran número de mujeres, pueden ser sometidas a un 
examen citológico de bajo costo y que puede permitir además, de la prevención del cáncer, el tratamiento de infecciones detectadas tanto por examen clínico, como por el estudio Papanicolaou.

Finalmente, queremos acotar que la Liga Peruana de Lucha contra el Cáncer, se fundó el 12 de noviembre de 1950, por un grupo de personas generosas que llamadas por su vocación de servicio al prójimo, decidieron fundar esta Institución, con la finalidad de prevenir el cáncer en la población peruana.

La Liga Peruana de Lucha contra el Cáncer, por ende, el Centro Detector del Cáncer en Tacna, es una institución privada que no recibe subvención estatal alguna, sus fuentes de ingreso son las colectas públicas anuales y las donaciones que generosamente recibe de las instituciones o personas con gran sensibilidad social.

Este trabajo no deja de tener pequeñas deficiencias, es consecuencia de la modesta colaboración de diversos profesionales, que lo hicieron en forma desinteresada.

\section{MATERIAL Y MÉTODOS}

\subsection{TIPO DE ESTUDIO}

Descriptivo y retrospectivo de mujeres que acudieron al Centro Detector del Cáncer en Tacna. ya sea a manera de consulta por uno o más sintomas; o por carecer de molestias, es decir, deseaban un despistaje.

\subsection{POBLACIÓN}

Mujeres que acuden voluntariamente o por campañas al Centro Detector del Cáncer en Tacna.

\subsection{RECOLECCIÓN DE DATOS}

\section{a) Selección de casos}

- Se trató de incluir el mayor número de casos, para obtener porcentajes aceptables, factibles de estudio y comentario. Se revisaron las historias clínicas del archivo del centro,sumando un total de 2,307 casos; pacientes que fueron vistas entre los años 1984 y 1993 (10 años).

Queremos resaltar en este punto, que el mayor número de pacientes acudieron en los primeros años, declinando su número en los últimos. Estimamos que este descenso se debe a que comenzaron a funcionar como centros de detección, otras instituciones relacionadas con la salud (postas médicas, hospitales, clínicas, etc).

Consideramos que las pacientes acudian a establecimientos cercanos a su domicilio. Lo expresado se puede apreciar en la siguiente relación:

\section{b) Recopilación de datos}

Se revisaron las historias clínicas, anotándose los datos en planillones confeccionados exprofesamente para nuestro propósito.

\section{c) Criterios de inclusión}

Consignamos los datos que consideramos necesarios para un estudio de conjunción de factores en el desarrollo de un cáncer ginecológico, especialmente de cuello uterino (el más frecuente).

Se incluyó:

- Edad de la paciente.

- Procedencia.

- Ocupación.

- Número de embarazos.

- Número de hijos.

- Número de abortos.

- Inicio de relaciones sexuales.

- Sintoma principal.

- Resultado citológico.

- Resultado microbiológico.

Algunos datos de importancia estadistica, pero la mayoria contribuyeron al estudio de factores predisponentes o determinantes en el desarrollo de la enfermedad.

\section{d) Criterios de exclusión}

- No se ha considerado el estado civil, pues por razones culturales comprensibles, en nuestro medio es frecuente el estatus de vida de la pareja como convivencia.

- Se ha descartado las historias clínicas cuya información ha sido muy pobre en datos.

\section{e) Definiciones}

Se utilizaron a manera de ilustración, algunas definiciones extraídas del diccionario 
Pschyrembel de Ginecologia y Obstetricia (ver bibliografía).

\section{f) Procesamiento de datos}

Las variables discretas fueron agrupadas en frecuencias y las continuas en rangos. Los datos recogidos fueron procesados con un ordenador personal, utilizando el programa de la hoja electrónica de microsoft word 2.0 y para la elaboración del texto se empleó el word perfect 6.0 .

\section{RESULTADOS}

1. El mayor número de pacientes referentes se encuentra entre los 20 y 40 años, edad en que la fertilidad y la vida sexual están en mayor actividad.

2. Predominan pacientes procedentes de Tacna, ya que el estudio se realizó en el centro detector de cáncer de esta ciudad. En segundo lugar, figuran pacientes procedentes de Puno.

3. La ocupación predominante de mujeres que asistieron al centro, es de amas de casa, en menor proporción, comerciantes, empleadas y estudiantes.

4. El número de embarazos se considera referencial como factor de alto riesgo. El mayor número presentó de 1 a 3 embarazos. También se considera apreciable (en segundo lugar) en mujeres que tuvieron de 4 a 6 embarazos.

5. El número de hijos se considera al igual que el punto anterior, como factor de alto riesgo: más de la mitad de pacientes estudiadas tenian entre 1 a 3 hijos. La cuarta parte tiene de 4 a 6 hijos. También figura un número regular de pacientes con más de 7 hijos.

6 . Respecto a los abortos (suponemos provocados la gran mayoría), se consideran como factor iniciador de infecciones o lesiones que por descuido pueden ir a la cronicidad; lesiones en el cuello uterino, sitio anatómico de alta frecuencia de neoplasias.

Aproximadamente la mitad de pacientes niegan abortos y la otra mitad han tenido uno o varios abortos.

7. Indudablemente que el coito precoz influye en la patogenia de cáncer de cuello uterino, un alto porcentaje refiere haberse iniciado sexualmente antes de los 20 años.

8. El sintoma principal es la leucorrea, coincide con los estudios comparados. Un poco más de la mitad de las mujeres estudiadas no presentaban sintomas.

9. Citológicamente la mitad de pacientes estudiadas presentaban algún tipo de infección (Grupo PAP II); un alto porcentaje sin alteración citológica y 34 casos con alguna displasia, que necesitaron estudio y tratamiento especial.

10. Considerando a las pacientes que se les demostró la presencia de microorganismos patógenos, encontramos que el primer lugar son triconomas, siguiéndole en orden decreciente gérmenes comunes, monilias, clamidias y finalmente gardanellas.

\section{DISCUSIÓN Y COMENTARIOS}

1. La detección entre mujeres supuestas sanas realizada por la Liga de Lucha Contra el Cáncer, a través del centro detector de Tacna, a pesar de tener mediano alcance, muestra de bondad del procedimiento. Si todas las mujeres se hicieran una prueba de papanicolaou anual, el cáncer cervical podría eliminarse como causa de muerte, desgraciadamente, menos de un $40 \%$ de las mujeres se hacen una prueba anual de papanicolaou.

2. Intentar desarrollar un plan de detección masiva de cáncer de cuello uterino en la Región, mediante el funcionamiento de un programa dirigido y financiado por el ministerio de Salud, en escala regional, utilizando unidades asistenciales ya existentes en forma escalonada: posta sanitaria, posta médica, centro de salud, hospital y otras instituciones.

3. Se debe organizar un plan educativo para los colegios y para toda la población del país mediante charlas, exhibición de peliculas, videos y distribución de folletos; así como el uso de medios de comunicación masiva: radio y televisión.

4. La preparación del personal médico, deberá ser constante y se efectuará mediante cursos de refrescamiento. Se debe contar con enfermeras y obstetrices debidamente entrenadas para la toma de muestras colpocervicales.

5. Debe conseguirse mediante la dación de una ley, la obligatoriedad del despistaje ginecológico para descubrir cáncer de cuello uterino, asi como de las lesiones premalignas. 
6. La biopsia debe ser obligatoria frente a una lesión sospechosa. El método se elegirá de acuerdo a cada caso (biopsia cervical con sacabocados, raspado endocervical, biopsia en cono con bisturi frío).

7. Si la paciente es joven, tiene enfermedad in situ y desea conservar su capacidad de tener hijos, la biopsia en cono conseguirá tanto confirmar su diagnóstico como proporcionar un tratamiento satisfactorio. No obstante, deben hacerse frotis repetidos de papanicolaou cada tres meses, para asegurar de que se ha eliminado toda la lesión y de que no recurre.

8. La prevención de la morbilidad y del cáncer cervical implica fundamentalmente el reconocimiento inicial y el tratamiento inmediato. Se debe reconocer a los factores de riesgo, es decir, la experiencia sexual y la promiscuidad, o la historia de displasia cervical. Debe continuarse el control de todas las mujeres postpúberes sobre una base anual regular, en especial en el caso de multiparas de los grupos socioeconómicos inferiores y de aquellas que son sexualmente activas.

9. Los métodos de higiene personal que pueden ayudar a prevenir el cáncer cervical incluyen la prevención y el tratamiento inmediato de la vaginitis y cervicitis: el lavado del pene antes de todo coito o el uso habitual de condones. Por supuesto, la abstinencia sexual constituye una medida efectiva aunque impracticable de profilaxis. Ciertamente, evitar la exposición sexual temprana y la promiscuidad, constituirian medidas eficaces.

\section{REFERENCIAS BIBLIOGRÁFICAS}

BARRIGA NORIEGA, Oscar. Detección de cáncer de cuello uterino. Tesis doctoral. Universidad Peruan Cayetano Heredia. Lima, Perú. 1973.

BENAVENTE LOZADA, Benjamín. Despistaje de cáncer del cuello uterino en el Hospital Militar Central. Tesis doctoral. Universidad Nacional Mayor de San Marcos. Lima, Perú. 1973.

BENSON C., Ralph. Diagnóstico y tratamiento ginecoobstétricos. México D.F. Quinta Edición. Ed. Manual Moderno. Pág. 872-887. 1989.

BENSON C., Ralph. Manual de ginecología y obstetrici a. México D.F. Séptima edición (Reimpresión). Ed. Manual Moderno. Pág. 519-530. 1992.

BERKOW ROBERT, M.D. TALBOTTH. JOHN, M.D. Manual Merck de diagnóstico y terapéutica. Rahwaym N.J. Sexta edición en español. Ed. Merck \& Sharp Dhme Research Laboratories. Pág. 989-991. 1978
CITOLOGÍA: Subdivisión y valoración de la citología ginecológica según Papanicolaou, y medidas diagnóstico - terapéuticas recomendadas en el ejemplo de la citología de la cérvix.

\begin{tabular}{|c|c|c|}
\hline GRUPO & CUADRO CELULAR & $\begin{array}{l}\text { VALORACIÓN CLINICAY } \\
\text { MEDIDAS RECOMENDADAS } \\
\text { RESPECTO AL CERVIX }\end{array}$ \\
\hline PAPI & Cuadro celular normal. & $\begin{array}{l}\text { Negativo: no es necesaria } \\
\text { medida alguna. }\end{array}$ \\
\hline PAP\| & $\begin{array}{l}\text { Cuadro celular normal con } \\
\text { modificaciones degenerativas } \\
\text { o regenerativas, inflamatorias } \\
\text { (agregaciones leucocitarias y } \\
\text { de microorganismos) y } \\
\text { metaplásicas. }\end{array}$ & $\begin{array}{l}\text { Negativo: control tras la } \\
\text { terapia (tratamiento del flujo. } \\
\text { hormonal). }\end{array}$ \\
\hline PAP III & $\begin{array}{l}\text { Cuadro celular poco claro, } \\
\text { condiconado por graves } \\
\text { modificaciones inflamatorias, } \\
\text { atróficas o degenerativas, o } \\
\text { por células intensamente } \\
\text { modificadas en sentido } \\
\text { regresivo. } \\
\text { Displasia leve moderada. }\end{array}$ & $\begin{array}{l}\text { Sospechoso frontis de control } \\
\text { en los } 3 \text { meses siguientes a la } \\
\text { terapia (tratamiento del flujo, } \\
\text { hormonas, eventualmente } \\
\text { electrocoagulación } \\
\text { conización). En caso de } \\
\text { células endometriales tras la } \\
\text { menopausia, abrasión. }\end{array}$ \\
\hline PAPIVa & $\begin{array}{l}\text { Células patológicas grave, } \\
\text { atipia celular): sospecha de } \\
\text { carcinoma (carcinoma in } \\
\text { situ). } \\
\text { Sospecha de } \\
\text { microcarcinoma; invasión } \\
\text { incipiente, cuestionable. }\end{array}$ & $\begin{array}{l}\text { Negativo: histologia (biopsia), } \\
\text { conización diagnóstica o } \\
\text { terapéutica, abrasión o, dado } \\
\text { el caso, histerectomia. }\end{array}$ \\
\hline PAPV & $\begin{array}{l}\text { Células tumorales, en masa, } \\
\text { claramente malignas, } \\
\text { sospecha elevada de } \\
\text { carcinoma invasivo. }\end{array}$ & $\begin{array}{l}\text { Positivo: urgente el } \\
\text { diagnóstico histológico. Si se } \\
\text { confirma el carcinoma, } \\
\text { aplicación de la terapia } \\
\text { quirúrgica, de medicina } \\
\text { nuclear o quimioterapia } \\
\text { oportunas. }\end{array}$ \\
\hline & $\begin{array}{l}\text { Preparado técnicamente } \\
\text { inutilizable }\end{array}$ & $\begin{array}{l}\text { Repetición del frontis en } 14 \\
\text { dias. }\end{array}$ \\
\hline
\end{tabular}

BONGIOVANNI ALFRED, M. Ginecología de la adolescente. Barcelona. Edición original. Ed. El Ateneo S.A. Pág. 125 $-141.1987$.

BOTERO JAIME, Jubiz H., Alfonso, HENAO GUILLERMO. Obstetricia y ginecología. Colombia. Cuarta edición. Ed. Carvajal S.A. Pág. 562 - 571. 1989

CAPLAN M., RONALD; J. SWEENEY I. WILLIAM. Avances en ginecologia y obstetricia. Barcelona. Edición española. Ed. Espaxs S.A. Pág. 543 - 553. 1982.

CUYLER HAMMOND, E. BURNS E.L. Detection of uterine cancer high and low risk groups. Revista Cancer 22, Pág. 1096-1107. 1968.

CHISTOPHERSON M., PARKER, J.E. Cervix cancer ande mass cytologic screening. Revista Cancer 26. Pág. 808 811. 1970. 